\title{
SINC APPROXIMATION OF CAUCHY-TYPE INTEGRALS OVER ARCS
}

\author{
FRANK STENGER ${ }^{1}$ \\ Dedicated to David Elliott on his 65th birthday
}

(Received 16 March 1998)

\begin{abstract}
In 1984, Elliott and Stenger wrote a joint paper on the approximation of Hilbert transforms over analytic arcs. In the present paper we sharpen the previously obtained results of Elliott and Stenger, and we also obtain formulas for approximating Cauchy integrals over analytic arcs.
\end{abstract}

\section{Introduction and summary}

David Elliott has made excellent contributions to the approximate solution of Cauchy singular integral equations (CSIE), to the approximation of Hilbert transforms, to quadrature, and to the approximation of functions, the majority of his work being his contributions to CSIE. During the past decade, CSIE have more and more frequently arisen in the solution of boundary layer integral equations, which stem mainly from partial differential equations. Accurate approximations of Hilbert transforms are required for the solution of CSIE, and Elliott has made several excellent contributions to this area $[3,14,16-18,20-28]$. His many excellent contributions to the solution of integral equations include $[1,2,4-10,13,15,16,19,22,27,29]$. While much of Elliott's excellent work is based on using polynomials as basis functions for approximation, in [27] Elliott and Stenger also wrote a paper on the approximation of Hilbert transforms and the solution of CSIE using Sinc methods as bases. Formulæ that are exact for polynomials converge rapidly in cases when the solution to the CSIE is smooth on the arc, whereas formulæ based on Sinc bases converge more rapidly in frequently

\footnotetext{
'Department of Computer Science, University of Utah, Salt Lake City, UT 841 12, USA.

(C) Australian Mathematical Society 2000, Serial-fee code 0334-2700/00
} 
occurring cases when the singular integral is analytic on interiors of the arc, but may have singularities at the end-points of the arc.

In many instances when the solution to the boundary layer equation has been determined, it is still necessary to obtain an analytic continuation of this solution to the interior of the domain. In the present paper we derive such "missing link" formulæ, for the case of Sinc approximation.

In Section 2 of the paper we shall review definitions and properties of Sinc approximation that are relevant for deriving the results. In Section 3, we derive Sinc-based formulæ and error bounds for approximation of both Hilbert and Cauchy transforms over rectifiable analytic arcs.

\section{Sinc spaces of approximation}

We shall describe here some relevant notions about approximation by Sinc methods.

Let $\mathscr{D}$ denote a simply-connected domain in the complex plane $\mathbb{C}$, let $1 \leq p \leq \infty$ and let $\mathbf{H}^{p}(\mathscr{D})$ denote the family of all functions $f$ that are analytic in $\mathscr{D}$, such that

$$
N_{p}(f, \mathscr{D}) \equiv \begin{cases}\left(\int_{\partial \mathscr{D}}|f(z)|^{p}|d z|\right)^{1 / p}<\infty & \text { if } 1 \leq p<\infty \\ \sup _{z \in \mathscr{D}}|f(z)|<\infty & \text { if } p=\infty .\end{cases}
$$

Let $\varphi$ denote a function which provides a conformal mapping of a simply connected domain $\mathscr{D}$ onto $\mathscr{D}_{d}$ where

$$
\mathscr{D}_{d}=\{w \in \mathbb{C}:|\Im w|<d\},
$$

with $d$ a fixed positive number. The function $\varphi$ is also a one-to-one map of $\Gamma$ onto $\mathbb{R}$, with $\Gamma$ an arc in $\mathscr{D}$, having end-points $a$ and $b$, so that $\varphi(a)=-\varphi(b)=-\infty$. The Sinc points are defined for $h>0$ and $k \in \mathbb{Z}$ by $z_{k}=\varphi^{-1}(k h)$.

Let us describe some simple Sinc spaces which go hand-in-hand with Sinc methods. Let $\alpha \in(0,1], \beta \in(0,1]$ and $0<d<\pi$. Let $\mathbf{M}_{\alpha, \beta}(\mathscr{D})$ denote the family of all functions $f \in \mathbf{H}^{\infty}(\mathscr{D})$, which have finite limits $f(a)$ and $f(b)$ at the end-points $a$ and $b$ of $\Gamma$, such that if we set

$$
\rho(z)=\exp (\varphi(z))
$$

then

$$
\begin{aligned}
& f(z)-f(a)=\mathscr{O}\left(|\rho(z)|^{\alpha}\right) \quad \text { as } \quad z \rightarrow a, \\
& f(z)-f(b)=\mathscr{O}\left(|\rho(z)|^{-\beta}\right) \text { as } z \rightarrow b,
\end{aligned}
$$


where the limits are taken with respect to $z$ from within $\mathscr{D}$. The notation $\mathbf{L}_{\alpha, \beta}(\mathscr{D})$ will be used for that subset of functions $f \in \mathbf{M}_{\alpha, \beta}(\mathscr{D})$ for which $f(a)=f(b)=0$.

Let $\mathscr{D}_{d}$ be defined as in (2.2), and for $0<d^{\prime}<d$, let $\varphi$ map $\mathscr{D}^{\prime}$ to $\mathscr{D}_{d^{\prime}}$. The most important properties of the spaces $\mathbf{L}_{\alpha, \beta}(\mathscr{D})$ and $\mathbf{M}_{\alpha, \beta}(\mathscr{D})$ are, in essence, that if $f \in$ $\mathbf{M}_{\alpha, \beta}(\mathscr{D})$, then $f^{\prime} / \varphi^{\prime} \in \mathbf{L}_{\alpha, \beta}\left(\mathscr{D}^{\prime}\right)$; if $f \in \mathbf{M}_{\alpha, \beta}(\mathscr{D})$, and if $\left(1 / \varphi^{\prime}\right)^{\prime}$ is uniformly bounded in $\mathscr{D}$, then $f^{(n)} /\left(\varphi^{\prime}\right)^{n} \in \mathbf{L}_{\alpha, \beta}\left(\mathscr{D}^{\prime}\right), n=1,2,3, \ldots$ Conversely, if $f^{\prime} / \varphi^{\prime} \in \mathbf{L}_{\alpha, \beta}(\mathscr{D})$, then $f \in \mathbf{M}_{\alpha, \beta}(\mathscr{D})$. For example, if $(a, b)$ is a finite interval, if $f$ is of class $\mathbf{L i p}_{\alpha}$ on $[a, b]$ and if $f$ is analytic in some region containing $(a, b)$, then $f \in \mathbf{M}_{\alpha, \alpha}(\mathscr{D})$.

\section{Sinc approximation}

Next, we define bases for Sinc approximation.

Let $N$ denote a positive integer, and let integers $M$ and $m$, a diagonal matrix $D(u)$ and an operator $V$ be defined as follows:

$$
\begin{aligned}
N & =\text { positive integer; } \\
M & =[\beta N / \alpha] \\
m & =M+N+1 ; \\
D(u) & =\operatorname{diag}\left[u\left(z_{-M}\right), \ldots, u\left(z_{N}\right)\right] ; \quad \text { and } \\
V(u) & =\left(u\left(z_{-M}\right), \ldots, u\left(z_{N}\right)\right)^{T},
\end{aligned}
$$

where [.] denotes the greatest integer function, $u$ is an arbitrary function defined on $\Gamma$ and " $T$ " denotes the transpose. Letting $\mathbb{Z}$ denote the set of all integers, set

$$
\begin{aligned}
\operatorname{sinc}(z) & =\frac{\sin (\pi z)}{\pi z}, \\
h & =\left(\frac{\pi d}{\beta N}\right)^{1 / 2}, \\
z_{j} & =\varphi^{-1}(j h), \quad j \in \mathbb{Z}, \\
\sigma_{j} & =\operatorname{sinc}\{[\varphi-j h] / h\}, \quad j=-M, \ldots, N, \\
w_{j} & =\sigma_{j}, \quad j=-M+1, \ldots, N-1, \\
w_{-M} & =\frac{1}{1+\rho}-\sum_{j=-M+1}^{N} \frac{1}{1+e^{j h}} \sigma_{j}, \\
w_{N} & =\frac{\rho}{1+\rho}-\sum_{j=-M}^{N-1} \frac{e^{j h}}{1+e^{j h}} \sigma_{j}, \\
\varepsilon_{N} & =N^{1 / 2} e^{-(\pi d \beta N)^{1 / 2}} .
\end{aligned}
$$


We remark that in the special case when we know a priori that $f$ vanishes at the left end-point $a$ of $\Gamma$, then it is more convenient to take $w_{-M}=\sigma_{-M}$; and similarly, we take $w_{N}=\sigma_{N}$ for the case when $f$ vanishes at the right end-point $b$ of $\Gamma$. Let us also define a row vector of basis functions,

$$
\mathbf{w}(z)=\left(w_{-M}(z), \ldots, w_{N}(z)\right),
$$

with $w_{j}$ defined as in (3.2).

Finally, we define a norm by

$$
\|f\|=\sup _{x \in \Gamma}|f(x)| .
$$

Next, we describe interpolation, definite and indefinite integration, and the approximation of Hilbert transforms via Sinc methods. In cases when proofs are omitted, they may be found in [30]; we shall, however, include proofs for the cases of Hilbert and Cauchy transforms.

3.1. Sinc interpolation Now we describe the basic procedure of Sinc interpolation.

THEOREM 3.1. If $f \in \mathbf{M}_{\alpha, \beta}(\mathscr{D})$, then as $N \rightarrow \infty$,

$$
\|f-\mathrm{w} V f\|=\mathscr{O}\left(\varepsilon_{N}\right) .
$$

3.2. Sinc collocation While the above defined Sinc basis completely determines the formulæ of Sinc approximation, the role of the basis is primarily in the evaluation, or plotting, of the final solution. This is the point of the theorem of this section, which enables us to reduce all Sinc computations to the solution of (linear or nonlinear) algebraic equations.

THEOREM 3.2. Let $\mathrm{c}=\left(c_{-M}, \ldots, c_{N}\right)^{T}$ be a vector of complex numbers, such that

$$
\left(\sum_{j=-M}^{N}\left|f\left(z_{j}\right)-c_{j}\right|^{2}\right)^{1 / 2}<\delta_{N},
$$

where $\delta_{N}$ is a positive number, depending only on $N$. If the conditions of Theorem 3.1 are satisfied then, as $N \rightarrow \infty$,

$$
\|f-\mathbf{w} \cdot \mathbf{c}\|=\mathscr{O}\left(\varepsilon_{N}+\delta_{N}\right) .
$$

3.3. Sinc quadrature Sinc quadrature approximation may be described as follows.

THEOREM 3.3. If $f / \varphi^{\prime} \in \mathbf{L}_{\alpha, \beta}(\mathscr{D})$, then, as $N \rightarrow \infty$,

$$
\left|\int_{\Gamma} f(x) d x-\left(V\left(h / \varphi^{\prime}\right)\right)^{T} V f\right|=\mathscr{O}\left(\varepsilon_{N}\right) .
$$

Indeed, it may be shown that by taking $h=(2 \pi d /(\beta N))^{1 / 2}$, the right-hand side of (3.8) may be replaced by $\varepsilon_{N}^{\sqrt{2}}$. 
3.4. Sinc indefinite integration For the case of Sinc indefinite integration and Sinc convolution, we need to determine matrices $A$ and $B$ of order $m$ as follows. Corresponding to an arbitrary integer $\ell$, we define $\delta_{\ell}^{(-1)}$ by

$$
\delta_{\ell}^{(-1)}=\int_{-\infty}^{\ell} \operatorname{sinc}(x) d x
$$

we form an $m \times m$ matrix $I^{(-1)}=\left[\delta_{i-j}^{(-1)}\right]$, with $\delta_{i-j}^{(-1)}$ denoting the $(i, j)^{t h}$ element of $I^{(-1)}$, and we then set $A=h I^{(-1)} D\left(1 / \varphi^{\prime}\right)$ and $B=h\left(I^{(-1)}\right)^{T} D\left(1 / \varphi^{\prime}\right)$.

THEOREM 3.4. If $f / \varphi^{\prime} \in \mathbf{L}_{\alpha, \beta}(\mathscr{D})$, then, as $N \rightarrow \infty$,

$$
\begin{aligned}
& \left\|\int_{a}^{x} f(t) d t-(\mathbf{w} A V f)(x)\right\|=\mathscr{O}\left(\varepsilon_{N}\right) \text { and } \\
& \left\|\int_{x}^{b} f(t) d t-(\mathbf{w} B V f)(x)\right\|=\mathscr{O}\left(\varepsilon_{N}\right) .
\end{aligned}
$$

3.5. Sinc convolution For the case of Sinc convolution, we define matrices $A$ and $B$ as for Sinc indefinite integration above, and in addition we shall also require the "Laplace transform",

$$
F(s)=\int_{E} e^{-t / s} f(t) d t, \quad E \supseteq(0,(b-a)),
$$

where the quotes indicate that we have replaced the $s$ in the usual Laplace transform with $1 / s$.

Our model integrals to be collocated are

$$
p(x)=\int_{a}^{x} f(x-t) g(t) d t \text { and } q(x)=\int_{x}^{b} f(t-x) g(t) d t,
$$

where $x \in \Gamma$.

In presenting these convolution results, we shall assume that $\Gamma=(a, b) \subseteq \mathbb{R}$. For Sinc collocation of $p$ and $q$, we assume that:

(1) The "Laplace transform" (3.11) exists for all $s$ such that $\Re s>0$;

(2) and (roughly-see [30] for more precise assumptions) that $p$ and $q$ belong to $\mathbf{M}_{\alpha, \beta}(\mathscr{D})$.

THEOREM 3.5. If the above assumptions are satisfied, and if $A$ and $B$ are defined as in the previous subsection, then, as $N \rightarrow \infty$,

$$
\|p-\mathbf{w} F(A) V g\|=\mathscr{O}\left(\varepsilon_{N}\right) \text { and }\|q-\mathbf{w} F(B) V g\|=\mathscr{O}\left(\varepsilon_{N}\right) .
$$


3.6. Cauchy and Hilbert transforms It is convenient here to present results derived in [27] along with newly derived results for Cauchy transforms.

We take $x \in \Gamma$ and $z \in \mathscr{D}^{+}$, with $\mathscr{D}^{+}$denoting the part of $\mathscr{D}$ to the right of $\Gamma$ as we traverse $\Gamma$ from $a$ to $b$, and we define the functions

$$
\begin{aligned}
s_{k}(x) & =\frac{h}{\pi} \frac{\sin \{\pi(\varphi(x)-k h) / h\}}{\varphi^{\prime}\left(z_{k}\right)\left(x-z_{k}\right)}, \\
t_{k}(x) & =\frac{h}{\pi i} \frac{\cos \{\pi(\varphi(x)-k h) / h\}-1}{\varphi^{\prime}\left(z_{k}\right)\left(x-z_{k}\right)} \text { and } \\
c_{k}(z) & =\frac{h}{2 \pi i} \frac{\exp \{i \pi(\varphi(z)-k h) / h\}-1}{\varphi^{\prime}\left(z_{k}\right)\left(z-z_{k}\right)} .
\end{aligned}
$$

Note that for $z=x$, we have $c_{k}(x)=\frac{1}{2}\left(s_{k}(x)+t_{k}(x)\right)$. If $k$ and $\ell$ are integers, we have

$$
\begin{aligned}
s_{k}\left(z_{\ell}\right) & = \begin{cases}0 & \text { if } k \neq \ell, \\
1 & \text { if } k=\ell,\end{cases} \\
t_{k}\left(z_{\ell}\right) & = \begin{cases}\frac{h}{\pi i} \frac{1-(-1)^{k-\ell}}{\varphi^{\prime}\left(z_{k}\right)\left(z_{k}-z_{\ell}\right)} & \text { if } k \neq \ell, \\
0 & \text { if } k=\ell \text { and }\end{cases} \\
c_{k}\left(z_{\ell}\right) & = \begin{cases}\frac{h}{2 \pi i} \frac{1-(-1)^{k-\ell}}{\varphi^{\prime}\left(z_{k}\right)\left(z_{k}-z_{\ell}\right)} & \text { if } k \neq \ell, \\
1 / 2 & \text { if } k=\ell .\end{cases}
\end{aligned}
$$

THEOREM 3.6. (a) If $g \in \mathbf{H}^{1}(\mathscr{D})$, then, for all $x \in \Gamma$, we have

$$
\begin{aligned}
\varepsilon_{1}(x) & \equiv g(x)-\sum_{k=-\infty}^{\infty} g\left(z_{k}\right) s_{k}(x) \\
& =\frac{\sin \left(\frac{\pi}{h} \varphi(x)\right)}{2 \pi i} \int_{\partial \mathscr{D}} \frac{g(\zeta)}{(\zeta-x) \sin \left(\frac{\pi}{h} \varphi(\zeta)\right)} d \zeta .
\end{aligned}
$$

(b) If $g \in \mathbf{H}^{1}(\mathscr{D})$, then, for all $x \in \Gamma$, we have

$$
\begin{aligned}
\varepsilon_{2}(x) & \equiv \frac{P . V .}{\pi i} \int_{\Gamma} \frac{g(t)}{t-x} d t-\sum_{k=-\infty}^{\infty} g\left(z_{k}\right) t_{k}(x) \\
& =\frac{1}{2 \pi} \int_{\partial \Phi} g(\zeta) \frac{\exp \left\{\frac{i \pi}{h} \varphi(\zeta) \operatorname{sgn} \Im \varphi(\zeta)\right\}-\cos \left(\frac{\pi}{h} \varphi(x)\right)}{(\zeta-x) \sin \left(\frac{\pi}{h} \varphi(\zeta)\right)} d \zeta
\end{aligned}
$$

(c) If $g \in \mathbf{H}^{1}(\mathscr{D})$, then, for all $z \in \mathscr{D}^{+}$, we have

$$
\varepsilon_{3}(z) \equiv \frac{1}{2 \pi i} \int_{\Gamma} \frac{g(\zeta)}{\zeta-z} d \zeta-\sum_{j=-\infty}^{\infty} g\left(z_{j}\right) c_{j}(z)
$$




$$
=\frac{1}{2 \pi i} \int_{\partial \mathscr{D}} g(\zeta) \frac{\exp \left\{\frac{i \pi}{h} \varphi(z)\right\}-\exp \left\{\frac{i \pi}{h} \varphi(\zeta) \operatorname{sgn} \Im \varphi(\zeta)\right\}}{(\zeta-z) \sin \left(\frac{\pi}{h} \varphi(\zeta)\right)} d \zeta
$$

We may note, for example, that in view of (3.18), (3.21) yields the approximation

$$
\left(\mathscr{S}_{g}\right)\left(z_{\ell}\right) \equiv \frac{\mathrm{P} . \mathrm{V} .}{\pi i} \int_{\Gamma} \frac{g(t)}{t-z_{\ell}} d t \approx \frac{h}{\pi i} \sum_{k \neq \ell} \frac{g\left(z_{k}\right)}{\varphi^{\prime}\left(z_{k}\right)} \frac{1-(-1)^{k-\ell}}{z_{k}-z_{\ell}} .
$$

For purposes of truncation of the above sums, we have the following theorem.

THEOREM 3.7. If $g \in H^{1}(\mathscr{D}), x \in \Gamma$ and $z \in \mathscr{D}^{+}$, then

$$
\begin{aligned}
\left|\varepsilon_{1}(x)\right| & \leq \frac{1}{2 \pi \sinh (\pi d / h)} N(g, \mathscr{D}, x), \\
\left|\varepsilon_{2}(x)\right| & \leq \frac{e^{-\pi d / h}+1}{2 \pi \sinh (\pi d / h)} N(g, \mathscr{D}, x) \text { and } \\
\left|\varepsilon_{3}(z)\right| & \leq \frac{e^{-\pi d / h}+1}{2 \pi \sinh (\pi d / h)} N(g, \mathscr{D}, z),
\end{aligned}
$$

where in each of the last three inequalities,

$$
N(g, \mathscr{D}, z)=\int_{\partial \mathscr{D}}\left|\frac{g(t)}{t-z} d t\right| .
$$

We remark that under our assumptions on $g$, the terms $N(g, \mathscr{D}, z)$ may become unbounded as $z$ approaches a point of $\partial \mathscr{D}$. In such a case the above sums must be interpreted in the sense of a relative error.

Of course, a uniform bound may be possible, that is, these equations will hold for all $z \in \mathscr{D}^{\prime}$, if $N(g, \mathscr{D}) \equiv \sup _{\left(z \in \mathscr{D}^{\prime}\right)} N(g, \mathscr{D}, z)<\infty$, with $\mathscr{D}^{\prime}$ defined at the end of Section 2 , and with $N(g, \mathscr{D}, z)$ defined as above. This was shown to be the case, for example, in [31] for several particular regions, for the case when $g \in \mathbf{L}_{\alpha, \beta}(\mathscr{D})$.

In addition, we have the bounds

$$
\sup _{x \in \Gamma}\left|s_{k}(x)\right| \leq e^{h}, \quad \sup _{x \in \Gamma}\left|t_{k}(x)\right| \leq e^{h} \quad \text { and } \quad \sup _{z \in \mathscr{Q}^{+}}\left|c_{k}(z)\right| \leq \frac{e^{h}}{\sqrt{2}},
$$

which are useful for bounding the error of approximation by a finite number of terms of the series (3.20)-(3.22).

The expressions for $\varepsilon_{1}(x)$ and $\varepsilon_{2}(x)$, as well as the above bounds on these terms, were established in [27]. We now prove the given results for $\varepsilon_{3}$.

PROOF. Proceeding similarly as in $\left[32\right.$, p. 247], we take $z \in \mathscr{D}^{+}$, and we start with the identity

$$
\frac{g(t)}{\varphi^{\prime}(t)} \frac{\varphi(t)-\varphi(z)}{t-z}-\sum_{j=-\infty}^{\infty} \frac{g\left(z_{j}\right)}{\varphi^{\prime}\left(z_{j}\right)} \frac{j h-\varphi(z)}{z_{j}-z} S(j, h) \circ \varphi(t)
$$




$$
=\frac{\sin \left(\frac{\pi}{h} \varphi(t)\right)}{2 \pi i} \int_{\partial \mathscr{D}} g(\zeta) \frac{\varphi(\zeta)-\varphi(z)}{(\varphi(\zeta)-\varphi(t))(\zeta-z) \sin \left(\frac{\pi}{h} \varphi(\zeta)\right)} d \zeta .
$$

We now multiply both sides of this equation by $\varphi^{\prime}(t) /[2 \pi i\{\varphi(t)-\varphi(z)\}]$, integrate with respect to $t$ over $\Gamma$, and use the identities

$$
\frac{1}{2 \pi i} \int_{\mathbb{R}} \frac{S(k, h)(t)}{t-z} d t=\frac{e^{i x(z-k h) / h}-1}{\frac{2 i \pi}{h}(z-k h)}, \quad \Im z>0
$$

and

$$
\frac{1}{2 \pi i} \int_{\mathbb{R}} \frac{\sin (\pi \xi / h)}{(\xi-w)(\xi-z)} d \xi=\frac{\exp \left\{\frac{i \pi}{h} w \operatorname{sgn} \Im w\right\}-\exp \left\{\frac{i \pi}{h} z \operatorname{sgn} \Im z\right\}}{2 i(w-z)}
$$

to deduce the expressions

$$
\begin{aligned}
E & \equiv \frac{1}{2 \pi i} \int_{\Gamma} \frac{g(t)}{t-z} d t-\sum_{j=-\infty}^{\infty} \frac{g\left(z_{j}\right)}{\varphi^{\prime}\left(z_{j}\right)} \frac{\exp \left\{\frac{i \pi}{h}(\varphi(z)-j h)\right\}-1}{\frac{2 \pi i}{h}\left(z-z_{j}\right)} \\
& =\frac{1}{2 \pi i} \int_{\partial \mathscr{D}} g(\zeta) \frac{\exp \left\{\frac{i \pi}{h} \varphi(z)\right\}-\exp \left\{\frac{i \pi}{h} \varphi(\zeta) \operatorname{sgn} \Im \varphi(\zeta)\right\}}{(\zeta-z) \sin \left(\frac{\pi}{h} \varphi(\zeta)\right)} d \zeta
\end{aligned}
$$

Now, in view of the inequalities

$$
\begin{gathered}
\left|\exp \left\{\frac{i \pi}{h} \varphi(\zeta) \operatorname{sgn} \Im \varphi(\zeta)\right\}\right|=\exp \left(-\frac{\pi d}{h}\right) \\
\left|\exp \left\{\frac{i \pi}{h} \varphi(z)\right\}\right| \leq 1 \text { and }\left|\sin \left(\frac{\pi}{h} \varphi(\zeta)\right)\right| \geq \sinh \left(\frac{\pi d}{h}\right)
\end{gathered}
$$

we get

$$
|E| \leq \frac{1+\exp (-\pi d / h)}{2 \pi \sinh (\pi d / h)} N(g, \mathscr{D}, z),
$$

where $N(g, \mathscr{D}, z)$ is defined as in (3.25). This establishes the inequality (3.24).

COROLlaRY 3.8. Let $\alpha$ and $\beta$ denote positive numbers such that $0<\alpha \leq 1$ and $0<\beta \leq 1$, let $g \in \mathbf{L}_{\alpha, \beta}(\mathscr{D})$, let $N$ be a positive integer, select $M$ as in (3.1) and $h$, $z_{j}$ and $\varepsilon_{N}$ as in (3.2). Let $\mathscr{D}^{+}$denote the part of $\mathscr{D}$ to the left of $\Gamma$ as we traverse $\Gamma$ from a to $b$. Let us assume that $N(g, \mathscr{D}, z) \leq N(g, \mathscr{D}), z \in \mathscr{D}^{\prime}$, with $\mathscr{D}^{\prime}$ defined as at the end of Section 2. Then there exist positive constants $C_{i}, i=1,2,3$, that are independent of $N$, such that

$$
\sup _{x \in \Gamma}\left|g(x)-\sum_{j=-M}^{N} g\left(z_{j}\right) s_{j}(x)\right|<C_{1} \varepsilon_{N}, \quad x \in \Gamma,
$$




$$
\begin{aligned}
& \sup _{x \in \Gamma}\left|\frac{P \cdot V \cdot}{\pi i} \int_{\Gamma} \frac{g(t)}{t-x}-\sum_{j=-M}^{N} g\left(z_{j}\right) t_{j}(x)\right|<C_{2} \varepsilon_{N}, \quad x \in \Gamma \quad \text { and } \\
& \sup _{z \in \mathscr{D}^{+}}\left|\frac{1}{2 \pi i} \int_{\Gamma} \frac{g(t)}{t-z}-\sum_{j=-M}^{N} g\left(z_{j}\right) c_{j}(z)\right|<C_{3} \varepsilon_{N}, \quad z \in \mathscr{D}^{\prime+} .
\end{aligned}
$$

PROOF. The proof is straightforward, using Theorem 3.6 and the inequalities (3.26). We omit the details.

Finally, we illustrate the application of Sinc convolution to the approximation of Cauchy integrals.

Since we can express the "Laplace transform" of the function $(2 \pi i(t-i y))^{-1}$ in terms of the exponential integral, that is,

$$
F(s) \equiv \frac{1}{2 \pi i} \int_{0}^{\infty} \frac{\exp (-t / s)}{t-i y} d t=\frac{1}{2 \pi i} \exp \left\{-\frac{i y}{s}\right\} E_{1}\left(-\frac{i y}{s}\right),
$$

we define a square matrix $A$ of order $m=M+N+1$ as in Section 3.4 , and if the assumptions of Corollary 3.8 are satisfied, we arrive at the approximation

$$
\frac{1}{2 \pi i} \int_{\Gamma} \frac{g(\zeta)}{\zeta-x-i y} d \zeta \approx \mathbf{w}(x) F(A) V g
$$

for which the relative error is $\mathscr{O}\left(\varepsilon_{N}\right)$ for every fixed $z=x+i y \in \mathscr{D}^{+}$. We have not yet made any numerical experiments on the use of this method for computation of Cauchy integrals.

\section{Acknowledgments}

Part of this research was carried out while the author was visiting the University of New South Wales during February and March of 1997. The hospitality of the UNSW and the support of the Australian Research Council are gratefully acknowledged. In addition, the author also gratefully acknowledges partial support of this work by NSF grant \# CCR-9307602, as well as the support of the University of Aizu, Japan, during the summer of 1997.

\section{References}

[1] D. Elliott, "A Chebyshev series method for the numerical solution of Fredholm integral equations", The Computer Journal 6 (1963) 102-111. 
[2] D. Elliott, "The approximate solution of singular integral equations", in Solution Methods for Integral Equations (ed. M. A. Golberg), (Plenum Publishing Corporation, 1979) 83-107.

[3] D. Elliott, "On the convergence of Hunter's quadrature rule for Cauchy principal value integrals", Nordisk Tidskrift für Informationsbehandlung 19 (1979) 457-462.

[4] D. Elliott, "Integral equations-ninety years on", in The Application and Numerical Solution of Integral Equations (eds R. S. Anderssen, F. R. De Hoog and M. A. Lukas), (Sijthoff and Noordhoff, 1980) 1-17.

[5] D. Elliott, "A convergence theorem for singular integral equations", J. Aust. Math. Soc. Series B 22 (1981) 539-552.

[6] D. Elliott, "Orthogonal polynomials associated with singular integral equations having a Cauchy kernel”, SIAM J. Math. Anal. 13 (1981) 59-66.

[7] D. Elliott, "The classical collocation method for singular integral equations", SIAM J. Numer. Anal. 19 (1982) 816-832.

[8] D. Elliott, "The numerical treatment of singular integral equations-a review", in Treatment of Integral Equations by Numerical Methods (eds C. T.H. Baker and G. F. Miller), (Academic Press, 1982) 297-312.

[9] D. Elliott, "Some aspects of singular integral equations-a numerical analyst's viewpoint", Contemporary Mathematics 9 (1982) 41-54.

[10] D. Elliott, "A Galerkin-Petrov method for singular integral equations", J. Aust. Math. Soc. (Series B) 25 (1983) 261-275.

[11] D. Elliott, "Rates of convergence for the method of classical collocation for solving singular integral equations", SIAM J. Numer. Anal. 21 (1984) 136-138.

[12] D. Elliott, "A comprehensive approach to the approximate solution of singular integral equations over the arc $(-1,1) "$, J. Integ. Eqs and Applics 2 (1989) 59-94.

[13] D. Elliott, "Convergence theorems for singular integral equations", in Numerical Solution of Integral Equations (ed. M. A. Golberg), (Plenum Press, 1990) 309-361.

[14] D. Elliott, "An asymptotic analysis of two algorithms for certain Hadamard finite-part integrals", IMA J. Num. Anal. 13 (1993) 445-462.

[15] D. Elliott, "Projection methods for singular integral equations", J. Integ. Eqs and Applics 2 (1995) 95-106.

[16] D. Elliott, "Three algorithms for Hadamard finite-part integrals and fractional derivatives", $J$. Comp. Appl. Math. 62 (1995) 267-283.

[17] D. Elliott and D. Delbourgo, "On the approximate evaluation of Hadamard finite-part integrals", IMA J. Num. Anal. 14 (1994) 219-233.

[18] D. Elliott and J. D. Donaldson, "On quadrature rules for ordinary and Cauchy principal value integrals over contours”, SIAM J. Numer. Anal. 14 (1977) 1078-1087.

[19] D. Elliott, I. K. Lifanov and G. S. Litvinchuk, "The solution in a class of singular functions of Cauchy type bisingular integral equations", J. Integ. Eqs and Applics to appear.

[20] D. Elliott and S. Okada, "The finite Hilbert transform in $L^{2 "}$, Math. Nachrichten 153 (1991) 43-56.

[21] D. Elliott and S. Okada, "Hölder continuous functions and the finite Hilbert transform", Math. Nachrichten 169 (1994) 219-233.

[22] D. Elliott and D. F. Paget, "An algorithm for the numerical evaluation of certain Cauchy principal value integrals", Numer. Math. 19 (1972) 373-385.

[23] D. Elliott and D. F. Paget, "On a convergence of a quadrature rule for evaluating certain Cauchy principal value integrals", Numer. Math. 23 (1975) 311-319.

[24] D. Elliott and D. F. Paget, "On a convergence of a quadrature rule for evaluating certain Cauchy principal value integrals; An addendum", Numer. Math. 25 (1976) 287-289.

[25] D. Elliott and D. F. Paget, "Gauss-type quadrature rules for Cauchy principal value integrals", Math. Comp. 33 (1979) 301-309. 
[26] D. Elliott and S. Prössdorf, "An algorithm for the approximate solution of integral equations of Mellin type", Numer. Math. 70 (1995) 427-452.

[27] D. Elliott and F. Stenger, "Sinc method of solution of singular integral equations", in IMACS Conference on CSIE, (Philadelphia, P.A., 1984), 155-166.

[28] D. Elliott and E. Venturino, "Sigmoidal transformations and the Euler-Maclaurin expansion for evaluating certain Hadamard finite-part integrals", Numer. Math. 77 (1997) 453-465.

[29] D. Elliott and W. G. Warne, "An algorithm for the numerical solution of linear integral equations", International Computation Center Bulletin 6 (1967) 207-224.

[30] M. Kowalski, K. Sikorski and F. Stenger, Selected Topics in Approximation and Computation (Oxford, 1995).

[31] F. Stenger, "Explicit, nearly optimal, linear rational approximations with preassigned poles", Math. Comp. 47 (1986) 225-252.

[32] F. Stenger, Numerical Methods Based on Sinc and Analytic Functions (Springer-Verlag, New York, 1993). 